\title{
On the structure of two-point Green-functions at next-to-leading order in $1 / N_{C}$
}

\author{
Juan José Sanz-Cillero \\ Department of Physics, Peking University, Beijing 100871, P.R. Chind
}

(Dated: July 5, 2017)

\begin{abstract}
The structure of the two-point QCD Green-functions is studied in this note in the limit of large number of colours. Their general form at next-to-leading order in $1 / N_{C}$ is derived keeping the infinite resonance summation and without relying on a particular realization of the hadronic action. It is found that the contributions from chiral operators without resonance fields of order $p^{4}$ or higher are irrelevant for the computation of the correlators and, hence, they can be dropped at the beginning of the calculation. The possibility of a more general cancelation of these local terms at the level of the generating functional is discussed.
\end{abstract}

PACS numbers: 11.15.Pg, 12.39.Fe, 11.55.Bq

\section{A large $-N_{C}$ hadronic field theory}

Quantum Chromodynamics (QCD) is greatly simplified in 't Hooft's large $-N_{C}$ limit [1, 2, 3]. Quark loops and non-planar topologies become suppressed and the theory can be then expressed in terms of tree-level hadron exchanges. In order to produce the perturbative QCD logarithms at deep euclidian momenta, an infinite number of narrow-width states is needed.

Meson loops are absent at large $-N_{C}$ and the amplitudes are analytic but for the real poles related to the narrow-width intermediate states. It is then possible to write down a lagrangian containing an infinite set of hadronic fields $\Phi_{k}$ of mass $M_{k}$ that do not interact each other when $N_{C} \rightarrow \infty$. In this note, we focus our attention on the meson sector, containing the Goldstone modes from the spontaneous chiral symmetry breaking and the mesonic resonances. The main assumption in the present work is that the vertex functions $\Gamma_{\Phi_{1} \ldots \Phi_{n}}^{(n)}$ can be described at the leading order in $1 / N_{C}$ (LO) by a finite number of hadronic operators. These must follow the expected scaling $N_{C}^{1-\frac{n}{2}}[3]$, although the $N_{C}$ dependence may not appear explicitly but hidden in the couplings $\lambda^{\Phi_{1} \ldots \Phi_{n}}$ of the corresponding operators.

As result of such resonance lagrangian, loops arise at subleading orders. The $N_{C}$-scaling of the operators in the LO action $S_{0}^{\mathrm{LO}}\left[\Phi_{k}, J\right]$ converts its loop expansion [4] into an expansion in powers of $1 / N_{C}$. The one-loop amplitudes contain ultraviolet (UV) divergences and nextto-leading order (NLO) counter-terms can be required to fulfill the renormalization.

This work studies the two-point correlators $\langle T\{\bar{q} \Gamma q(x) \bar{q} \Gamma q(0)\}\rangle$ or combinations of them, where $\bar{q} \Gamma q$ refers to some vector or axial-vector current, or to scalar or pseudo-scalar densities. These Green-functions are provided by the corresponding scalar functions $\Pi(s)$ and their short-distance behaviour can be derived through the operator product expansion (OPE) [5]. The aim of this article is to show the structure of UV divergences and counter-terms of $\Pi(s)$ at $\mathrm{NLO}$ in $1 / N_{C}$. In order to avoid any model dependence, the infinite tower of states is kept all along the paper. Likewise, no explicit realization of the lagrangian is assumed, just the existence of such hadronic action.

Phenomenological large- $N_{C}$ analyses have found that some $\mathcal{O}\left(p^{4}\right)$ chiral operators without resonance fields are absent from the hadronic lagrangian both at LO and at NLO in $1 / N_{C}$ [ 6 ]. We will often refer along the paper to the chiral order $\mathcal{O}\left(p^{m}\right)$ of the operators in the way of the chiral counting, with $p^{m}$ denoting the number or derivatives or external auxiliary fields $v^{\mu}, a^{\mu}, s, p$ [7, 8]. This note proves that even if the presence of these chiral operators is needed to renormalize some vertex functions, they turn out to be irrelevant for the calculation of $\Pi(s)$ and do not have physical content [9]. The correlators are shown to be fully described in terms of the couplings in the $\mathrm{LO}$ action. A change in $\alpha_{s}$ would modify the hadronic parameters but $\Pi(s)$ would be still determined by the operators in $S_{0}^{\mathrm{LO}}\left[\Phi_{k}, J\right]$; new operators could arise at NLO but the correlator can be completely recovered even if we ignore the value of these new couplings.

The functions $\Pi(s)$ are analytical in the variable $s$ out of the positive real $s$-axis and they accept general complex variable techniques $10,11,[12,13]$. Based on the asymptotic behaviour at $|s| \rightarrow \infty$, prescribed by the OPE, one can write $m$-subtracted dispersion relations for $m \geq n$, where $n$ denotes the minimal number of subtractions required for $\Pi(s)$. Other quantities with these properties are the two-meson form factors that vanish at short distances and accept unsubtracted dispersion relations [14, 15, 16]. Nevertheless, they bring further complications on other aspects and they will not be discussed here.

\section{Moments and $n$-subtracted relations}

For the Green-function analysis, we define a set of moments $\mathcal{A}_{\Pi}^{(m)}(s)$, which can be related to the spectral func- 
tion for $m \geq n[13]$ :

$\mathcal{A}_{\Pi}^{(m)}(s) \equiv \frac{(-s)^{m}}{m !} \frac{d^{m}}{d s^{m}} \Pi(s)=\int \frac{(-s)^{m} d t}{(t-s)^{m+1}} \frac{1}{\pi} \operatorname{Im} \Pi(t)$.

The moments with $m<n$ can be derived through the recursive relation

$$
\frac{\mathcal{A}_{\Pi}^{(m-1)}(s)}{s^{m-1}}=\frac{\mathcal{A}_{\Pi}^{(m-1)}\left(s_{0}\right)}{s_{0}^{m-1}}-m \int_{s_{0}}^{s} \frac{d t}{t^{m}} \mathcal{A}_{\Pi}^{(m)}(t) .
$$

In order to fix $\Pi(s)=\mathcal{A}_{\Pi}^{(0)}(s)$ one needs to provide the value of $n$ subtraction constants, $\left\{\mathcal{A}_{\Pi}^{(m)}\left(s_{0}\right)\right\}_{m=0}^{n-1}$ at some energy $s_{0}$.

In some cases, the OPE may prescribe $\Pi(s) \stackrel{s \rightarrow-\infty}{\longrightarrow} 0$ but the unsubtracted dispersive relation leads to an infinite resonance summation $\Pi(s)=\sum_{\Phi_{k}} \Pi_{\Phi_{k}}(s)$ that is badly defined [17, 18]. For instance, the unsubtracted dispersion relation for the $\mathrm{SS}+\mathrm{PP}$ correlator is not convergent. Hence, the infinite resonance summation in $\Pi(s)_{\mathrm{SS}-\mathrm{PP}}$ is not absolutely convergent [17]. One needs to perform a number of subtractions $n$ higher than what is expected just from OPE arguments. Once the infinite series has been summed up in $\mathcal{A}_{\Pi}^{(n)}(s)$, one can use Eq. (2) to recover the lower moments $\left\{\mathcal{A}_{\Pi}^{(m)}(s)\right\}_{m=0}^{n-1}$. The choice $s_{0}=-\infty$ gives $\mathcal{A}_{\Pi}^{(m)}\left(s_{0}\right)=0$ for the two-point Greenfunctions that are chiral order-parameters [5] and fixes $\Pi(s)$.

The Green-functions that are not chiral orderparameters are more cumbersome. The subtraction constants can be obtained from the renormalized perturbative QCD correlator $\Pi\left(s_{0}\right)^{\mathrm{pQCD}}$ and its moments $\mathcal{A}_{\Pi}^{(m)}\left(s_{0}\right)^{\mathrm{pQCD}}$ at deep euclidian momentum $-s_{0} \gg \Lambda_{\mathrm{QCD}}^{2}$. Furthermore, $\mathcal{A}_{\Pi}^{(m)}\left(s_{0}\right)$ is a finite and pure QCD quantity for $m \geq n$ that can be fully determined in the resonance theory. Its matching to perturbative QCD allows to recover $\alpha_{s}$ in terms of the hadronic parameters [18]. In the chiral limit, provided the value of the strong coupling constant and one renormalization condition for $\Pi\left(s_{0}\right)^{\mathrm{PQCD}}$ (not fixed by QCD), the rest of moments $\mathcal{A}_{\Pi}^{(m)}\left(s_{0}\right)^{\mathrm{pQCD}}$ turns out to be completely determined. This demonstrates that all the QCD information of $\Pi(s)$ is actually contained in $\mathcal{A}_{\Pi}^{(n)}(s)$. Moreover, this moment, determined within the resonance $1 / N_{C}$ framework, yields the evolution from $s_{0}$ in the perturbative QCD regime down to any energy.

Before entering into the calculation of $\Pi(s)$, some more preliminary definitions are needed. Let us consider a general function $f(t)$, analytical in the whole complex plane except for logarithmic branches and single and double poles at $t=M_{k}^{r}{ }^{2}$. If $f(t)$ accepts a $n$-subtracted dispersive relation, one has the mathematical identity for its moment,

$$
\begin{aligned}
\mathcal{A}_{f}^{(n)}(s) & =\Delta \mathcal{A}_{f}^{(n)}(s) \\
& +\sum_{k}\left[\frac{(-s)^{n} Z_{k}^{f}}{\left(M_{k}^{r}{ }^{2}-t\right)^{n+1}}+\frac{(n+1)(-s)^{n} D_{k}^{f}}{\left(M_{k}^{r 2}-t\right)^{n+2}}\right],
\end{aligned}
$$

with the coefficients

$$
\begin{aligned}
D_{k}^{f} & =\lim _{t \rightarrow M_{k}^{r} 2} \operatorname{Re}\left\{\left(t-M_{k}^{r 2}\right)^{2} f(t)\right\}, \\
Z_{k}^{f} & =-\lim _{t \rightarrow M_{k}^{r 2}} \operatorname{Re}\left\{\frac{d}{d t}\left[\left(t-M_{k}^{r 2}\right)^{2} f(t)\right]\right\} .
\end{aligned}
$$

and the absorptive contribution

$$
\begin{aligned}
\Delta \mathcal{A}_{f}^{(n)}(s) & =\lim _{\epsilon \rightarrow 0^{+}}\left\{\frac{1}{\pi} \int_{\mathcal{R}_{\epsilon}} \frac{d t(-s)^{n} \operatorname{Im} f(t)}{(t-s)^{n+1}}\right. \\
& \left.-\frac{2}{\pi \epsilon} \sum_{k} \lim _{t \rightarrow M_{k}^{r} 2} \frac{(-s)^{n}\left(t-M_{k}^{r 2}\right)^{2} \operatorname{Im} f(t)}{(t-s)^{n+1}}\right\},
\end{aligned}
$$

with $\mathcal{R}_{\epsilon}=[0,+\infty)-\bigcup_{k}\left(M_{k}^{r}{ }^{2}-\epsilon, M_{k}^{r}{ }^{2}+\epsilon\right)$. The function $\Delta \mathcal{A}_{f}^{(n)}(s)$ only depends on $\operatorname{Im} f(t)$ at $t \neq M_{k}^{r}{ }^{2}$.

For sake of clarity, the explicit derivation is shown for correlators obeying unsubtracted dispersion relations. In order to get the $n$-subtracted expressions, one should make the replacements,

$$
\begin{aligned}
\Pi(s) & \longrightarrow \mathcal{A}_{\Pi}^{(n)}(s) \\
\frac{1}{M_{k}^{r 2}-s} & \longrightarrow \frac{(-s)^{n}}{\left(M_{k}^{r 2}-s\right)^{n+1}}, \\
\frac{1}{\left(M_{k}^{r 2}-s\right)^{2}} & \longrightarrow \frac{(n+1)(-s)^{n}}{\left(M_{k}^{r 2}-s\right)^{n+2}}, \\
\sum_{j \geq 0} c_{j} s^{j} & \longrightarrow \sum_{j \geq n} \frac{(-1)^{n} j !}{n !(j-n) !} c_{j} s^{j} .
\end{aligned}
$$

\section{Large $-N_{C}$ amplitude}

In the large $N_{C}$ limit, the absorptive part of $\Pi(t)$ is composed by a series delta functions,

$$
\frac{1}{\pi} \operatorname{Im} \Pi(t)=\sum_{k} Z_{k} \delta\left(t-M_{k}^{2}\right)
$$

with $Z_{k}$ and $M_{k}$ being, respectively, the residues and the pole positions of the QCD amplitude at large $-N_{C}$. Using dispersive relations, one gets the form of the LO amplitude,

$$
\Pi(s)=\sum_{k} \frac{Z_{k}}{M_{k}^{2}-s} .
$$

This amplitude can be described through the tree-level diagrams of a lagrangian with local operators containing the meson fields $\Phi_{k}$ of mass $M_{k}$ [19]. Depending on the 
realization of the hadronic lagrangian, the leading action may contain $\mathcal{O}\left(p^{2}\right)$ resonance operators that give the resonance exchange [20],

$$
\Pi(s)_{\mathrm{res}}=\sum_{k} \frac{F_{k}^{2}}{M_{k}^{2}-s} .
$$

Notice that if $\Pi(s)$ refers to the difference of two correlators, the summation may contain $(-1)$ factors.

There may also be some local contributions of the form

$$
\Pi(s)_{\mathrm{loc}}=\sum_{j} c_{j} s^{j} .
$$

These operators are provided by chiral perturbation theory if we work within a chiral invariant framework [8]. Some terms only depend on the external fields, e.g., $v_{\mu}$ and $a_{\mu}$ in the case of the $\mathcal{O}\left(p^{4}\right)$ operator $H_{1} \mathcal{O}_{H_{1}}$ [8]. Nevertheless, there can be chiral invariant operators, like $L_{10} \mathcal{O}_{L_{10}}$ at $\mathcal{O}\left(p^{4}\right)$, that contain Goldstone fields in addition to the contact terms with only external sources [8]. They are non-trivial and contribute to other processes $\mathcal{M}_{\Pi}$ related to $\Pi(s)$ through chiral symmetry.

One may also think of further resonance operators contributing at tree-level. At LO, the only bilinear operators are the canonical kinetic term, e.g., $-\Phi_{k}^{\dagger}\left(\partial^{2}+M_{k}^{2}\right) \Phi_{k}$ for spin-0 particles. On the other hand, there can be resonance operators contributing to the $\bar{q} \Gamma q \rightarrow \Phi_{k}$ vertices with a number of derivatives higher than $\mathcal{O}\left(p^{2}\right)$. A term like this would give the amplitude

$$
\Pi(s)_{\mathrm{res}^{\prime}}=\frac{\lambda s^{j+1}}{M_{k}^{2}-s} .
$$

This can be always rewritten in the form

$$
\Pi(s)_{\mathrm{res}^{\prime}}=\frac{\lambda\left(M_{k}^{2}\right)^{j+1}}{M_{k}^{2}-s}+\sum_{j^{\prime}} c_{j^{\prime}}^{\lambda} s^{j^{\prime}} .
$$

Hence, these operators reproduce the same structures found before in Eqs. (9) and (10) and do not contain further independent information. The equations of motion (EoM) provide a deeper understanding of this simplification. Every spin-0 resonance obeys a classical EoM of the form $\partial^{2} \Phi_{k}=-M_{k}^{2} \Phi_{k}+\chi[J, \pi]+\mathcal{O}\left(\Phi^{2}\right)$, where $\chi[J, \pi]$ stands for operators containing only external sources and Goldstone fields [16, 23]. The operators linear on $\Phi_{k}$ and contributing to $\bar{q} \Gamma q \rightarrow \Phi_{k}$ can be fully transformed thanks to the EoM into operators without resonance fields and terms that do not contribute to $\Pi(s)$. The case of particles with spin different from zero is more complicate due to the appearance of Lorentz structures in the EoM. Nevertheless, the study of vector fields in the antisymmetric tensor formalism casts a similar result [16, 21]. In any case, all that matters for this paper is that any tree-level contribution to $\Pi(s)$ at LO in $1 / N_{C}$ can be written in the form of Eqs. (9) and (10), where $F_{k}$,
$M_{k}, c_{j}$ carry all the independent information. These EoM simplifications will be also crucial in the NLO analysis.

Finally, matching the QFT and the dispersive expression in Eq. (8), one gets

$$
F_{k}^{2}=Z_{k}, \quad c_{j}=0 .
$$

This can be better understood through the example of the vector correlator. If the spin-1 field are realized in the Proca formalism, there are no $\mathcal{O}\left(p^{2}\right)$ resonance operators [22]. The lowest order is $\mathcal{O}\left(p^{3}\right)$ and the correlator results

$$
\Pi_{\mathrm{VV}}(s)=\sum_{k} \frac{f_{V_{k}}^{2} s}{M_{V_{k}}^{2}-s}+c_{0}+c_{1} s+\ldots
$$

It can be then recasted in the form of Eqs. (9) and (10),

$$
\Pi_{\mathrm{VV}}(s)=\sum_{k} \frac{f_{V_{k}}^{2} M_{V_{k}}^{2}}{M_{V_{k}}^{2}-s}+c_{0}^{\prime}+c_{1}^{\prime} s+\ldots
$$

with $c_{0}^{\prime}=c_{0}-\sum_{k} f_{V_{k}}^{2}$ and $c_{j}^{\prime}=c_{j}$ in the rest of cases. The dispersion relation sets $c_{j}^{\prime}=0$ and the correlator becomes determined just in terms of $M_{V_{k}}$ and $Z_{k}=f_{V_{k}}^{2} M_{V_{k}}^{2}$, that contain all the physical information of $\Pi(s)$ :

$$
\Pi_{\mathrm{VV}}(s)=\sum_{k} \frac{f_{V_{k}}^{2} M_{V_{k}}^{2}}{M_{V_{k}}^{2}-s} .
$$

The infinite summation in the $c_{j}^{\prime}$ does not present a problem. To regularize it, one can always construct a series of theories with a finite number of states containing the same couplings $f_{V_{k}}, M_{V_{k}}$ but with $c_{j}$ chosen such that $c_{j}^{\prime}=0$. In the limit when one includes the infinite tower of resonance, such theories recover the structure of the correlator in Eq. (8) prescribed by the dispersion relations.

\section{Amplitude at next-to-leading order in $1 / N_{C}$}

At NLO, a shift in the lagrangian parameters is required in order to absorb the one-loop UV divergences. The renormalization of the $\mathcal{O}\left(p^{2}\right)$ resonance couplings, $M_{k}^{2}=M_{k}^{r 2}+\delta M_{k}^{2}, F_{k}^{2}=F_{k}^{r 2}+\delta F_{k}^{2}$ gives,

$$
\Pi(s)_{\mathrm{res}}=\sum_{k}\left[\frac{F_{k}^{r 2}+\delta F_{k}^{2}}{M_{k}^{r 2}-s}-\frac{F_{k}^{r 2} \delta M_{k}^{2}}{\left(M_{k}^{r 2}-s\right)^{2}}\right] .
$$

Although the analysis of some phenomenological lagrangians have found that the corresponding local operators $c_{j} \mathcal{O}_{c_{j}}$ vanish from the action at NLO [6], we will consider the more general case when $c_{j} \neq 0$ and a nonzero contribution from $\Pi(s)_{\text {loc }}$, given in Eq. (10), arises at NLO in $1 / N_{C}$.

Higher $\mathcal{O}\left(p^{m}\right)$ operators linear in $\Phi_{k}$ can be removed at $\mathrm{NLO}$ in the same way as it was done at LO in Eqs. (11) 
and (12). In addition, one may have at NLO in $1 / N_{C}$ tree-level contributions to the resonance self-energies. A generic operator of this kind would yield the amplitude

$$
\Pi(s)_{\mathrm{res}^{\prime}}=\frac{\lambda s^{j+2}}{\left(M_{k}^{r 2}-s\right)^{2}},
$$

where $M_{k}^{2}$ and $M_{k}^{r}{ }^{2}$ can be used indistinctly at NLO. This can be reexpressed as

$\Pi(s)_{\text {res }^{\prime}}=\frac{\lambda\left(M_{k}^{r 2}\right)^{j+2}}{\left(M_{k}^{r 2}-s\right)^{2}}-(j+2) \frac{\lambda\left(M_{k}^{r 2}\right)^{j+1}}{M_{k}^{r 2}-s}+\sum_{j^{\prime}} c_{j^{\prime}}^{\lambda} s^{j^{\prime}}$,

which reproduces the momentum structures in $\Pi(s)_{\text {res }}$ and $\Pi(s)_{\text {loc. }}$. Therefore, it does not provide any extra independent information to the correlator. Again, the study of the leading order action EoM gives a deeper understanding to this kind of simplifications [16, 21, 23]. Hence, any tree-level contribution to $\Pi(s)$ will be expressed in the form of $\Pi(s)_{\text {loc }}$ and $\Pi(s)_{\text {res }}$ in Eqs. (10) and (17).

The two-point Green-functions are provided at the one-loop level by the one-particle irreducible topologies (1PI) shown in Fig.(1). Hence, the perturbative expression of $\Pi(t)$ contains single an double poles at $t=M_{k}^{r}$. The one-loop amplitude is given by the oneand two-point Feynman integrals, respectively $A_{0}\left(M^{2}\right)$ and $B_{0}\left(s, M^{2}, M^{\prime 2}\right)$ [24]. Any term proportional to $A_{0}\left(M^{2}\right)$ is a rational functions of the form

$$
\Pi(s)_{A_{0}}=\sum_{j} \Gamma_{j}^{A_{0}} s^{j}+\sum_{k}\left[\frac{Z_{k}^{A_{0}}}{M_{k}^{r 2}-s}+\frac{D_{k}^{A_{0}}}{\left(M_{k}^{r^{2}}-s\right)^{2}}\right] .
$$

The contribution from a given $\Phi_{1} \Phi_{2}$ absorptive cut is provided by the $B_{0}\left(s, M_{\Phi_{1}}^{2}, M_{\Phi_{2}}^{2}\right)$ Feynman integral through the term

$$
\Pi(s)_{\Phi_{1} \Phi_{2}}=\xi_{\Phi_{1} \Phi_{2}}(s) \cdot \mathcal{F}_{\Phi_{1} \Phi_{2}}(s)^{2} \cdot B_{0}\left(s, M_{\Phi_{1}}^{2}, M_{\Phi_{2}}^{2}\right),
$$

where $\mathcal{F}_{\Phi_{1} \Phi_{2}}(s)$ is the $\Phi_{1} \Phi_{2}$ form-factor at large- $N_{C}$ and the kinematical factor $\xi_{\Phi_{1} \Phi_{2}}(s)$ is a known polynomial that depends on the channel under consideration [15, 16]. For instance, one has $\xi_{\pi \pi}(s)=\frac{2}{3}$ for the vector correlator. The form-factor, and more exactly the combination $\xi_{\Phi_{1} \Phi_{2}}(t) \mathcal{F}_{\Phi_{1} \Phi_{2}}(t)^{2}$, provides the $\Phi_{1} \Phi_{2}$ contribution to the spectral function. Hence, if the correlator follows a $n-$ subtracted dispersion relation, also does the expression in Eq. (21). It can be then expressed through the master relation in Eq. (3),

$\Pi(s)_{\Phi_{1} \Phi_{2}}=\Delta \Pi(s)_{\Phi_{1} \Phi_{2}}+\sum_{k}\left[\frac{Z_{k}^{\Phi_{1} \Phi_{2}}}{M_{k}^{r 2}-s}+\frac{D_{k}^{\Phi_{1} \Phi_{2}}}{\left(M_{k}^{r 2}-s\right)^{2}}\right]$,

where $\Delta \Pi(s)_{\Phi_{1} \Phi_{2}}$ is determined by $\operatorname{Im} \Pi(s)_{\Phi_{1} \Phi_{2}}$ at $t \neq M_{k}^{r 2}$ and it is therefore free of $\mathrm{UV}$-divergences.

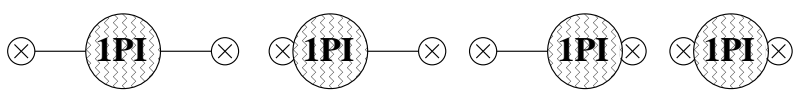

FIG. 1: 1PI contributions to the one-loop correlators.

These are contained in the coefficients $Z_{k}^{\Phi_{1} \Phi_{2}}, D_{k}^{\Phi_{1} \Phi_{2}}$, defined as in Eq. (4). Notice that for sake of simplicity we have written down the expression for $\Pi(s)$. The transcription to higher moments $\mathcal{A}_{\Pi}^{(n)}$ through Eq. (6) is left to the reader. Putting the different one-loop diagrams together one gets the structure,

$$
\begin{aligned}
\Pi(s)_{1 \ell} & =\Delta \Pi(s)_{1 \ell}+\sum_{j} \Gamma_{j}^{1 \ell} s^{j} \\
& +\sum_{k}\left[\frac{Z_{k}^{1 \ell}}{M_{k}^{r 2}-s}+\frac{D_{k}^{1 \ell}}{\left(M_{k}^{r 2}-s\right)^{2}}\right] .
\end{aligned}
$$

Since the only two-meson absorptive contribution to the correlator comes from the one-loop amplitudes, one can write $\Delta \Pi(s)$ instead of $\Delta \Pi(s)_{1 \ell}$. The summation of the $\Gamma_{j}, Z_{k}, D_{k}$ from the different loop diagrams in $\Pi(s)_{A_{0}}$ and $\Pi(s)_{\Phi_{1} \Phi_{2}}$ yields, respectively, the constants $\Gamma_{j}^{1 \ell}, Z_{k}^{1 \ell}$, $D_{k}^{1 \ell}$. Whereas the derivation of the different terms in Eq. (23) is straight-forward in the case of a QFT with a finite number of states, its definition is more cumbersome in the infinite resonance limit. This discussion is relegated for the end of the section, after the main result is obtained.

Summing up the one-loop amplitude $\Pi(s)_{1 \ell}$, the local terms $\Pi(s)_{\text {loc }}$ and the resonance exchanges $\Pi(s)_{\text {res }}$, one gets the correlator up to NLO:

$$
\begin{gathered}
\Pi(s)=\Delta \Pi(s)+\sum_{j}\left[c_{j}+\Gamma_{j}^{1 \ell}\right] s^{j} \\
+\sum_{k}\left[\frac{Z_{k}}{M_{k}^{r 2}-s}+\frac{D_{k}}{\left(M_{k}^{r 2}-s\right)^{2}}\right],
\end{gathered}
$$

with

$$
\begin{aligned}
& Z_{k}=F_{k}^{r 2}+\delta F_{k}^{2}+Z_{k}^{1 \ell}, \\
& D_{k}=-F_{k}^{r}{ }^{2} \delta M_{k}^{2}+D_{k}^{1 \ell} .
\end{aligned}
$$

The coefficients $Z_{k}$ and $D_{k}$ contain UV divergences from $\Pi(s)_{1 \ell}$ that are canceled through the renormalizations

$$
\begin{aligned}
\delta F_{k}^{2} & =-Z_{k}^{1 \ell}+\Delta Z_{k}, \\
F_{k}^{r}{ }^{2} \delta M_{k}^{2} & =D_{k}^{1 \ell}-\Delta D_{k},
\end{aligned}
$$

where the renormalization scheme for the couplings and masses is set by the finite constants $\Delta Z_{k}$ and $\Delta D_{k}$, respectively. For instance, one can choose the on-shell scheme $\Delta Z_{k}=\Delta D_{k}=0$.

Since the correlator $\Pi(s)$ obeys a $n$-subtracted dispersion relation (here shown unsubtracted for sake of clarity), one can apply the identity in Eq.(3):

$$
\Pi(s)=\Delta \Pi(s)+\sum_{k}\left[\frac{Z_{k}}{M_{k}^{r 2}-s}+\frac{D_{k}}{\left(M_{k}^{r 2}-s\right)^{2}}\right] .
$$


The comparison to the QFT calculation in Eq. (24) leads to the identities

$$
\begin{aligned}
F_{k}^{r 2}+\Delta Z_{k} & =Z_{k}, \\
\Delta D_{k} & =D_{k}, \\
c_{j}+\Gamma_{j}^{1 \ell} & =0 .
\end{aligned}
$$

Provided a renormalization scheme $\Delta Z_{k}, \Delta D_{k}$, the renormalized couplings $F_{k}^{r}$ are given by the residues of the QCD amplitude at NLO in $1 / N_{C}$. The polynomial contribution vanishes identically and it is zero for any energy, being $c_{j}$ and $\Gamma_{j}^{1 \ell}$ irrelevant for the calculation of $\Pi(s)$ (or the moment $\mathcal{A}_{\Pi}^{(n)}(s)$ in the case of $n$-subtracted dispersion relations). The analysis of the $S S-P P$ correlator in the single resonance approximation provides a practical example on the use of these techniques [15, 16].

However, the irrelevance of these local terms in other amplitudes $\mathcal{M}_{\Pi}$ related to $\Pi(s)$ by chiral symmetry is not so trivial. In the case when $\Gamma_{j}^{1 \ell}=0$, the parameters $c_{j}$ become zero and the local operators $\mathcal{O}_{c_{j}}$ disappear from the hadronic action. This situation has been found for some matrix elements within an approximation of large$N_{C}$ QCD, where only spin-0 states were included and $S_{0}^{\mathrm{LO}}\left[\Phi_{k}, J\right]$ contained operators with at most two derivatives [6]. On the other hand, a non-zero value of $\Gamma_{j}^{1 \ell}$ induces a coupling $c_{j} \neq 0$ and the operators $c_{j} \mathcal{O}_{c_{j}}$ could, at first, add some analytical contribution to other chirally related amplitudes $\mathcal{M}_{\Pi}$.

A last important detail is whether the summation of infinite diagrams is well defined (as it happens for a theory with a finite number of states). In order to regularize this limit, it is possible to follow a procedure similar to that one employed at LO. One may consider the correlator $\Pi(s)^{\text {part. }}$ provided by the partial summation of a finite number of one-loop diagrams. For every partial sum one can always keep $F_{k}^{r}$ and $M_{k}^{r}$ fixed and reproducing the residues $Z_{k}$ and pole positions of the full QCD amplitude, and with the $c_{j}$ couplings chosen such that $c_{j}=-\Gamma_{j}^{1 \ell, \text { part. }}$. In the limit when one sums an infinite number of diagrams such theories lead to the Greenfunction prescribed by dispersion theory in Eq. (27).

\section{Structure of the generating functional}

To end with, a digression on the importance of the local terms in other amplitudes is presented. We examine the generating functional $W[J]$, given in terms of chiral invariant operators that preserve the QCD symmetries [8, 16, 23]. On what follows we will refer only to spin- 0 correlators. Hadrons with higher spins complicate the argumentation since one may find that there are no $\mathcal{O}\left(p^{2}\right)$ resonance operators, as it happens with the spin-1 fields in the Proca formalism 22].

At leading order, $W[J]$ is provided by the LO action $S_{0}^{\mathrm{LO}}\left[\Phi_{k}^{c l}, J\right]$, with $\Phi_{k}^{c l}$ the classical meson fields that de- pend implicitly on the external sources $J$. Actually, the LO analysis showed that the Green-functions are determined by the operators of lowest chiral order: $\mathcal{O}\left(p^{2}\right)$ for the scalar correlator [20], $\mathcal{O}\left(p^{3}\right)$ for the vector correlator in the Proca formalism [22]...

Following the derivation of the NLO amplitude in the note, the one-loop generating functional shows the structure

$$
\begin{aligned}
W[J]= & S_{0}^{\mathrm{LO}}\left[\Phi_{k}^{c l}, J\right]+S_{1}^{\mathrm{LO}}\left[\Phi_{k}^{c l}, J\right] \\
& +\sum_{j} c_{j} \int d x^{d} \mathcal{O}_{c_{j}}\left[\pi^{c l}, J\right]+[\ldots],
\end{aligned}
$$

where [...] stands for an irrelevant constant and operators that do not enter in the calculation of $\Pi(s)$ or chirally related amplitudes $\mathcal{M}_{\Pi}$. The term $S_{1}^{\mathrm{LO}}\left[\Phi_{k}^{c l}, J\right]$ is the one-loop contribution to the generating functional coming from the integration of the quadratic fluctuations of $\Phi_{k}$ in the action $S_{0}^{\mathrm{LO}}\left[\Phi_{k}, J\right]$ around $\Phi_{k}^{c l}[16,23]$. The local terms $\mathcal{O}_{c_{j}}$ do not contain resonance fields and only depend on external sources and Goldstone fields $\pi^{c l}$.

Although we have implicitly considered $n$-subtracted dispersion relations all along the paper, let us suppose that the $\Pi(s)$ accept unsubtracted dispersion relations. It is then possible to write the decomposition,

$$
\begin{aligned}
S_{1}^{\mathrm{LO}}\left[\Phi_{k}^{c l}, J\right] & =\bar{S}_{1}^{\mathrm{LO}}\left[\Phi_{k}^{c l}, J\right] \\
& +\sum_{j} \Gamma_{j}^{1 \ell} \int d x^{d} \mathcal{O}_{c_{j}}\left[\pi^{c l}, J\right]+[\ldots],
\end{aligned}
$$

where $\bar{S}_{1}^{\mathrm{LO}}\left[\Phi_{k}^{c l}, J\right]$ generates the absorptive contribution $\Delta \Pi(s)$ and the UV divergent terms $Z_{k}^{1 \ell}, D_{k}^{1 \ell}$. These are renormalized by the $\mathcal{O}\left(p^{2}\right)$ resonance operators in $S_{0}^{\mathrm{LO}}\left[\Phi_{k}^{c l}, J\right]$. The polynomial divergences of $\Pi(s)$ are contained in the constants $\Gamma_{j}^{1 \ell}$. Putting the contributions $S_{0}^{\mathrm{LO}}\left[\Phi_{k}^{c l}, J\right]$ and $S_{1}^{\mathrm{LO}}\left[\Phi_{k}^{c l}, J\right]$ together with the $\Pi(s)$ constraint $c_{j}=-\Gamma_{j}^{1 \ell}$ one gets the one-loop functional,

$$
W[J]=\bar{S}_{0}^{\mathrm{LO}}\left[\Phi_{k}^{c l}, J\right]+\bar{S}_{1}^{\mathrm{LO}}\left[\Phi_{k}^{c l}, J\right]+[\ldots] .
$$

We have written here $\bar{S}_{0}^{\mathrm{LO}}\left[\Phi_{k}^{c l}, J\right]$ since only the $\mathcal{O}\left(p^{2}\right)$ operators in $S_{0}^{\mathrm{LO}}\left[\Phi_{k}^{c l}, J\right]$ are relevant for $\Pi(s)$ at tree-level. Hence, the replacement $W[J] \longrightarrow \bar{W}[J]$ leaves unchanged the part of the generating functional under study. This operation is understood in the sense of removing any operator in $W[J]$ of order higher than $p^{2}$ (contributing to $\Pi(s)$ or $\left.\mathcal{M}_{\Pi}\right)$. This removes the local terms $\mathcal{O}_{c_{j}}\left[\pi^{c l}, J\right]$ that produce the polynomial contributions in correlator. The separation of the $\Gamma_{j}^{1 \ell}$ is not arbitrary since these are the only local operators in $S_{1}^{\mathrm{LO}}\left[\Phi_{k}^{c l}, J\right]$ of order $p^{4}$ or higher. This means that any local contribution $c_{j}$ or $\Gamma_{j}^{1 \ell}$ appearing in the generating functional $W[J]$, in the amplitudes $\Pi(s)$ or in related processes $\mathcal{M}_{\Pi}$ can be directly truncated and removed from the computation at the very beginning. 
After the transformation into $\bar{W}[J]$, one reaches a functional that generates the two-point Green-functions and is renormalizable; a NLO shift of the parameters in the $\mathcal{O}\left(p^{2}\right)$ LO action $\bar{S}_{0}^{\mathrm{LO}}\left[\Phi_{k}^{c l}, J\right]$ is enough to absorb the UV divergences of the one-loop contribution $\bar{S}_{1}^{\mathrm{LO}}\left[\Phi_{k}^{c l}, J\right]$.

Although this argumentation only considers spin-0 correlators and assumes unsubtracted dispersion relations, it provides an interesting insight. The local terms $\Gamma_{j}^{1 \ell}$, generated by the one-point Feynman integrals $A_{0}\left(M^{2}\right)$, may depend on the realization of the hadronic lagrangian 20, 25, 26]. However, this arbitrariness is not reflected in the physical amplitudes, which are uniquely determined in terms of the two-meson absorptive contributions $\operatorname{Im} \Pi(t)_{\Phi_{1} \Phi_{2}}$ and the resonance couplings $F_{k}^{r}$ and masses $M_{k}^{r}$. Likewise, it is important to recall that on the contrary to what happens in momentum space, the QCD correlators are finite in configuration space and no problem of definition should arise in the large $-N_{C}$ amplitudes. For instance, large $-N_{C}$ QCD holographic models do not find problems on handling the infinite tower of states in the five-dimensional configuration space but the infinite summations of Kaluza-Klein modes in momentum space may eventually become ill-defined [27]. Further investigations along these lines are relegated to future works.

\section{Conclusions}

The present note demonstrates that the moments $\mathcal{A}_{\Pi}^{(n)}(s)$ are determined in QCD at NLO in $1 / N_{C}$ by the operators in $S_{0}^{\mathrm{LO}}\left[\Phi_{k}, J\right]$, which give the two-meson absorptive contributions $\operatorname{Im} \Pi(t)_{\Phi_{1} \Phi_{2}}$ and the renormalized couplings $F_{k}^{r}$ and $M_{k}^{r}$. These two quantities provide the residues $Z_{k}$ of the QCD matrix element and the position of the poles at NLO in perturbation theory. Moreover, it is shown that the full correlator $\Pi(s)$ is actually determined by the same parameters as $\mathcal{A}_{\Pi}^{(n)}(s)$ together with one renormalization condition $\Pi\left(s_{0}\right)$ in the case of divergent QCD correlators.

In a general effective field theory, the NLO amplitude is determined by new NLO terms in the action. This analysis suggests that a large- $N_{C}$ resonance QFT should not be regarded as an effective theory. In the case of two-point Green-functions, it rather resembles a "renormalizable theory" up operators $c_{j} \mathcal{O}_{c_{j}}[\pi, J]$ containing at most Goldstone fields and external sources. The local couplings $c_{j}$ eventually cancel the one-loop terms $\Gamma_{j}^{1 \ell}$ and become irrelevant for the amplitude, which can be computed even if their value remains unknown. Hence, they can be dropped from the calculation at the very beginning.

\section{Acknowledgments}

I would like to thank S. Peris, A. Pich, I. Rosell and P. Ruiz-Femenía for their criticisms and comments on the manuscript. The ideas in the present work were partially developed at IPN-Orsay and during the visit to $\mathrm{ECT}^{*}$ on July 2006. This work is supported in part by National Nature Science Foundations of China under contract number 10575002,10421503

* Electronic address: cillero@th.phy.pku.edu.cn

[1] G. 't Hooft, Nucl. Phys. B 72 (1974) 461.

[2] G. 't Hooft, Nucl. Phys. B 75 (1974) 461.

[3] E. Witten, Nucl. Phys. B 160 (1979) 57.

[4] L.H. Ryder, Quantum Field Theory, Cambridge University Press (1985); A. Das, Field Theory, a Path Integral Approach, World Scientific (1993).

[5] M.A. Shifman et al., Nucl. Phys. B 147 (1979) 385.

[6] J. Portolés et al. arXiv:hep-ph/0611375.

[7] S. Weinberg, Physica 96A (1979) 327.

[8] J. Gasser and H. Leutwyler, Ann. Phys. 158 (1984) 142; Nucl. Phys. B 250 (1985) 465.

[9] A similar thing happens with the operators $\beta_{1} \mathcal{O}_{\beta_{1}}, \beta_{2} \mathcal{O}_{\beta_{2}}$ in chiral perturbation theory, which are proportional to the equations of motion. See for instance T.E. Rudy et al., Phys. Rev. C 50 (1994) 447-459.

[10] L.D. Landau, Nucl. Phys. 13 (1959) 181.

[11] S. Weinberg, Phys. Rev. Lett. 18 (1967) 507;

[12] S.L. Adler, Phys. Rev. D 10 (1974) 3714.

[13] E. de Rafael arXiv:hep-ph/9802448.

[14] G.P. Lepage and S.J. Brodsky, Phys. Lett. B 87 (1979) 359; Phys. Rev. D 22 (1980) 2157; 24 (1981) 1808.

[15] I. Rosell et al. arXiv:hep-ph/0610290; J.J. Sanz-Cillero arXiv:hep-ph/0610304.

[16] I. Rosell arXiv:hep-ph/0701248.

[17] M. Golterman and S. Peris, Phys. Rev. D 74 (2006) 096002.

[18] J.J. Sanz-Cillero, Nucl. Phys. B 732 (2006) 136-168.

[19] C.G. Callan et al., Phys. Rev. 177 (1969) 2247; S.R. Coleman et al., Phys. Rev. 177 (1969) 2239;

[20] G. Ecker et al., Nucl. Phys. B 321 (1989) 311; V. Cirigliano et al., Nucl. Phys. B 753 (2006) 139.

[21] I. Rosell et al., JHEP 0408 (2004) 042.

[22] G. Ecker et al., Phys. Lett. B 223 (1989) 425.

[23] I. Rosell et al., JHEP 0512 (2005) 020.

[24] G. Passarino and M. J. G. Veltman, Nucl. Phys. B 160 (1979) 151.

[25] J. F. Donoghue et al., Phys. Rev. D 39 (1989)1947.

[26] M. Bando et al., Phys. Rev. Lett. 54 (1985) 1215; M. Harada and K. Yamawaki, Phys. Lett. B 297 (1992) 151 ;

[27] J. Hirn and V. Sanz arXiv:hep-ph/0702005]. 